\title{
Prognostic value of simple frailty and malnutrition screening tools in patients with acute heart failure
}

Short title: frailty and malnutrition in HF

Word count: abstract (241 words); text - excluding references and legends to figures

(2521 words). Number of references: 39.

S.Sze, MBBS; $;^{1,2}$ J. Zhang, PhD; ${ }^{1}$ P. Pellicori, MD; ${ }^{1}$ D. Morgan, MD ${ }^{2}$ A. Hoye, PhD; ${ }^{1}$ A.L. Clark, MA, MD. ${ }^{1}$

${ }^{1}$ Department of Cardiology, Castle Hill Hospital, Hull York Medical School (at University of Hull), Kingston upon Hull, HU16 5JQ, UK

${ }^{2}$ Department of Cardiology, Diana, Princess of Wales Hospital, Grimsby, Northeast Lincolnshire, DN33 2BA, UK.

Corresponding author: Shirley Sze

Department of Cardiology,

Hull York Medical School

Hull and East Yorkshire Medical Research and Teaching Centre

Castle Hill Hospital, Cottingham, Kingston upon Hull, HU16 5JQ, UK

Tel: + 441482461811

Fax: +44 1482461779

Email: Shirley.sze@nhs.net

\section{Conflict of interest: None.}




\begin{abstract}
Background: Frailty and malnutrition are common in patients with heart failure (HF), and are associated with adverse outcomes. We studied the prognostic value of three malnutrition and three frailty indices in patients admitted acutely to hospital with HF.
\end{abstract}

Methods: 265 consecutive patients (62\% males, median age 80 (interquartile range (IQR): 72-86) years, median NTproBNP 3633 (IQR: 2025-6407) ng/l) admitted with HF between 2013 and 2014 were enrolled. Patients were screened for frailty using the Derby frailty index (DFI), acute frailty network (AFN) frailty criteria and clinical frailty scale (CFS), and for malnutrition using the geriatric nutritional risk index (GNRI), controlling nutritional status (CONUT) score and prognostic nutritional index (PNI).

Results: According to the CFS (>4), DFI and AFN; 53\%, 50\% and 53\% were frail, respectively. According to the GNRI ( $\leq 98)$, CONUT score ( $>4)$ and PNI $(\leq 38), 46 \%, 46 \%$ and $42 \%$ patients were malnourished, respectively.

During a median follow-up of 598 days (IQR 319-807 days), 113 patients died. One year mortality was $1 \%$ for those who were neither frail nor malnourished; $15 \%$ for those who were either malnourished or frail; and 65\% for those who were both malnourished and frail. Amongst the malnutrition scores, PNI, and amongst the frailty scores, CFS, increased model performance most compared with base model. A final model including CFS and PNI increased c-statistic for mortality prediction from 0.68 to 0.84 . 
Conclusion: Worsening frailty and malnutrition indices are strongly related to worse outcome in patients hospitalised with HF.

Keywords: frailty, malnutrition, heart failure, mortality, score 


\section{Introduction}

Admission to hospital for heart failure (HF) is very common, ${ }^{1}$ as is subsequent re-admission. ${ }^{2}$ Not only is hospitalisation expensive, each admission is associated with a worse prognosis. ${ }^{3}$ Modern medical therapy for patients with chronic HF is based on the results from large, randomised clinical trials, yet the patients included in trials often poorly reflect the reality of patients in clinical practice. Around 25\% of patients admitted to hospitals with HF are older than 80 years. ${ }^{4}$ Elderly patients with HF usually have complex comorbidities and clinical features distinct from subjects enrolled in major clinical trials conducted in HF. Amongst these, frailty ${ }^{5}$ and malnutrition ${ }^{6}$ are two very common features in patients with HF which are often overlooked. $7,8,9,10,11$

Screening HF patients for malnutrition and frailty might be helpful in identifying patients at high risk of re-admission who might benefit from tailored treatments or interventions to help reduce risk. ${ }^{12,13}$ Although there are several simple screening tools for malnutrition and frailty in patients with a variety of diseases, ranging from cancer to other chronic conditions, 141516 171819202122 there is no consensus as to which tool is best in patients with HF. Amongst nutritional scores, the COntrolling NUTritional Status index $(\mathrm{CONUT})^{18}$, the prognostic nutritional index (PNI) ${ }^{21}$ and the geriatric nutritional risk index (GNRI) ${ }^{19}$ are the most widely studied in HF populations. ${ }^{6}$ The clinical frailty scale (CFS $)^{20}$ is popular and widely used while the Derby frailty index (DFI) ${ }^{23}$ and the acute frailty network (AFN) frailty criteria ${ }^{24}$ are simple frailty identification tools developed recently.

We aimed to investigate the relation between nutrition, frailty and outcome amongst patients admitted to hospital for HF. 


\section{Methods}

\section{Study population}

This is a study of 265 consecutive patients admitted to a district hospital (Diana, Princess of Wales Hospital) in Grimsby, United Kingdom, between January 2013 and December 2014 with HF secondary to left ventricular systolic dysfunction (LVSD) were enrolled in the study. LVSD was defined as a left ventricular ejection fraction (LVEF) $<40 \%$ at echocardiography, or at least moderate LVSD on visual inspection on an echocardiogram.

All patients had a full medical history, physical examination and blood tests within a few hours of admission. An echocardiogram was performed within the first 24 hours from admission by an experienced sonographer using a Vivid 5 Scanner (GE, Fairfield, Connecticut) with a 2.5-MHz phased-array transducer. LVEF was calculated using Simpson’s method. Height and weight were measured on the first day of admission. Weight was measured with the patients in their nightwear and without their shoes. Body mass index (BMI) was calculated using the formula: BMI = weight in kilograms / (height in meters) squared.

The study was approved by the research and development department at Diana, Princess of Wales Hospital, Grimsby, UK.

\section{Frailty indices}

Patients were screened for frailty using three indices. (Appendix 1) Each subject was assessed by a physician who reviewed the medical and nursing notes together with any 
assessments performed by the multidisciplinary healthcare team including physiotherapists, occupational therapists, dieticians and pharmacists.

The Derby frailty index (DFI; scored as frail vs non-frail), is a quick pragmatic frailty identification tool initially developed in $2013 .{ }^{23} \mathrm{~A}$ patient is classified as frail if one of the following criteria was met: 1) >65 years old and a care home resident; 2) >75 with confusion, falls or reduced mobility; 3) $>84$ years old with $>4$ co-morbidities. ${ }^{23}$

The acute frailty network criteria (AFN; scored as frail vs non-frail) defines frailty as present in (a) people aged $85+$ or (b) people aged 65+ with one or more of the following presenting features: cognitive impairment; resident in a care home; history of fragility fractures; Parkinson's disease; recurrent falls. ${ }^{24}$

The clinical frailty scale (CFS; measured between 1 (very fit) and 9 (terminally ill). Subjects are scored according to their functional capacity, level of dependence and comorbidities. For example, a patient with uncontrolled symptoms who is not frankly dependent is classified as vulnerable and scores 4 on the CFS; while an individual with limited dependence on others for instrumental activities of daily living including finances, transportation, heavy housework and medications will be classified as mildly frail and scores 5 on the CFS. Subjects with a CFS $>4$ are classified as frail. ${ }^{20}$

We also measured comorbidity using the Charlson comorbidity index/score. ${ }^{25}$

\section{Malnutrition scores}


Patients were screened for malnutrition using 3 indices (Appendix 2). Malnutrition scores were calculated for each patient based on biochemical variables and anthropometric measurements.

The geriatric nutritional risk index (GNRI; measured between 65-138) screens for malnutrition suing serum albumin level and the ratio of body weight to ideal body weight. ${ }^{19}$ We calculated ideal weight using the formula: height in centimetres $(\mathrm{H})-100-[(\mathrm{H}-150) / 4]$ for men and H-100-[(H-150)/2.5] for women. ${ }^{19}$ GNRI is calculated using the formula: [1.489 $\mathrm{x}$ albumin $(\mathrm{g} / \mathrm{L})]+[41.7 \times$ current weight/ ideal weight $] .{ }^{19}$ Patients with GNRI $>98$ are not malnourished, those with GNRI 92-98, 82-91, $<82$ are at mild, moderate and severe risk of malnutrition, respectively. Subjects with GNRI $\leq 98$ are classified as malnourished.

The COntrolling NUTritional Status index (CONUT score; measured between 0-12), was developed by Ignacaio de Ulibarri and colleagues in 2005 as a screening tool for assessment of nutritional status of inpatients. ${ }^{18}$ The CONUT score uses serum albumin, cholesterol and total lymphocyte count. Patients with a CONUT score 0-1 have normal nutritional status, those with CONUT score 2-4, 5-8, 9-12 are at mild, moderate and severe risk of malnutrition respectively. Subjects with CONUT score $>4$ are classified as malnourished.

The prognostic nutritional index (PNI; measured between 22-70) is another nutritional screening tool, calculated using the formula: $10 \mathrm{x}$ serum albumin $(\mathrm{g} / \mathrm{dL})+0.005 \mathrm{x}$ total lymphocyte count $\left(\mathrm{mm}^{3}\right) .{ }^{21}$ Individuals are classified as normal (PNI $\left.>38\right)$, at moderate (PNI 35-38) or severe $(\mathrm{PNI}<35)$ risk of malnutrition. Subjects with $\mathrm{PNI} \leq 38$ are classified as malnourished.

\section{End points and follow-up}


Patients were followed until the $1^{\text {st }}$ of February 2016 and the primary end point was all-cause mortality. Mortality was ascertained by using medical records (updated systematically onto a NHS electronic database), autopsy reports and death certificates.

\section{Statistical analysis}

Continuous variables are expressed as a median with interquartile range (IQR) and categorical variables are expressed as percentages. Pearson's correlation coefficients with scatter plots were used to assess the correlations between two variables. Venn diagrams were used to illustrate the relationship between indices.

The relation between a variable and outcome was explored using Cox regression analysis. The assumptions of Cox regression were tested. Log-transformation was applied when the data were very right-skewed. We used two base models. To assess malnutrition scores, the base model included age, sex, haemoglobin, atrial fibrillation, log NTproBNP, creatinine, sodium, recurrent falls and the presence of ischaemic heart disease. To assess the frailty scores, the base model included sex, haemoglobin, atrial fibrillation, log NTproBNP, creatinine, sodium and the presence of ischaemic heart disease. Age and recurrent falls were excluded as they are part of the DFI and AFN frailty criteria. We added each of the frailty and malnutrition scores in turn to the base model and used c-statistics to evaluate model discrimination in survival analysis. The frailty and malnutrition scores with the highest cstatistics were used to construct a final model for predicting mortality. Using the best frailty and malnutrition indices, we stratified our cohort into 4 groups: frail and malnourished; frail but not malnourished; malnourished but not frail; and neither malnourished nor frail. KaplanMeier curves were constructed to compare survival between the 4 groups. 
Statistical analysis was carried out using Stata 14 and SPSS 23 software packages. The twotailed level of statistical significance was set at $\mathrm{p}<0.05$.

\section{$\underline{\text { Results }}$}

Baseline characteristics of the cohort are shown in Table 1.

The majority of patients enrolled was male (62\%) and elderly (median age with IQR: 80 (7286) years); 74\% had NYHA class III or IV symptoms; and median NTproBNP was 3633 (IQR: (2025-6407) ng/l. Around half of the cohort was 'frail' or 'malnourished'. According to the CFS (>4), DFI and AFN; 53\%, 50\% and 53\% were frail, respectively; 43\% (N=113) were classified as frail by all 3 frailty indices (Figure 1). According to the GNRI ( $\leq 98)$, CONUT score $(>4)$ and PNI ( $\leq 38), 46 \%, 46 \%$ and $42 \%$ patients were malnourished, respectively; 30\% ( $\mathrm{N}=79)$ were classified as malnourished by all 3 malnutrition indices (Figure 2).

\section{Relationship between malnutrition, frailty and clinical data}

Frail and malnourished patients were older; more likely to be nursing home residents; more likely to suffer from recurrent falls, dementia, anaemia and atrial fibrillation; had lower BMI, worse symptoms and renal function; and were less likely to be on an angiotensin converting enzyme inhibitor/ angiotensin receptor blocker or a beta blocker. (Table 1) 
Frailty and malnutrition indices correlated with each other (Table 2). Figure 3 shows the relationship between CFS and PNI. Although increasing frailty correlated with worsening malnutrition, the correlation was weak $\left(\mathrm{R}^{2}=0.22, \mathrm{p}<0.001\right)$.

\section{Malnutrition and frailty scores: associations with mortality}

During a median follow-up of 598 days (interquartile range 319- 807 days), 113 patients died. Univariable predictors of mortality are shown in table 3. Worsening frailty and malnutrition were both associated with worse outcome.

Of the variables that were significant in univariable analysis (excluding 3 frailty variables), 3 were significant predictors in a multivariable model including malnutrition scores (Table 3).

Of the variables that were significant in univariable analysis (excluding 3 nutritional variables, age and recurrent falls, which are included in AFN and DFI), 2 were significant predictors in a multivariable model for frailty scores (Table 3).

Addition of malnutrition and frailty indices to the base models both increased model performance for mortality prediction (Table 4). Amongst the malnutrition scores, PNI, and amongst the frailty scores, CFS, increased model performance most compared with base model.

Of the variables that were significant in univariable analysis, only PNI and CFS were significant predictors in the final survival model (Table 5). Adding both CFS and PNI to the base model including sex, haemoglobin, atrial fibrillation, log NTproBNP, creatinine, sodium, recurrent falls and the presence of ischaemic heart disease, had a c-statistic of 0.84 . Patients who were frail and malnourished had an almost 30 times greater mortality risk than those who were neither frail nor malnourished (Figure 4). Mortality at 1 year was 65\% for 
those who were frail and malnourished; $15 \%$ for those who were either frail or malnourished and only $1 \%$ for those who were neither frail nor malnourished.

\section{$\underline{\text { Discussion }}$}

We have found that malnutrition and frailty are both very common amongst patients admitted to hospital with heart failure. The prevalence of malnutrition and frailty in our cohort was around $45 \%$ and 50\% respectively, similar to findings from other studies (34\% to $70 \%{ }^{6,26,27}$ for malnutrition and $30 \%$ to $50 \%{ }^{28}$ for frailty). Ours is the first paper to compare directly several different indices of malnutrition and frailty in patients hospitalised with HF. Whilst we found that there was substantial overlap between the patients identified as either frail or malnourished by each index, the overlap was not absolute. Although we found a correlation between increasing frailty and worsening malnutrition, the relation was weak: only around $20 \%$ of the variation was due to variation in the other. This finding suggests that frailty and malnutrition, despite having overlapping features, are distinct entities.

We found that both malnutrition and frailty are strongly related to outcome in patients hospitalised with HF. In our final multivariate survival model, only CFS and PNI were significant predictors of mortality. These indices eliminate all other variables as potential indicators of outcome, presumably because they include many aspects of other potential variables in a composite score.

Malnutrition and cachexia in HF arise not only as a direct consequence of HF but also from mechanisms common to other chronic disease states. Firstly, patients with HF have a higher basal metabolic rate than normal. ${ }^{11}$ Secondly, gut oedema and hepatic congestion may result in nausea, early satiety and reduced food absorption. ${ }^{11}$ HF is a chronic inflammatory state which induces metabolic disturbances. ${ }^{11}$ Impaired cardiac pump function and subsequent 
hypoperfusion of peripheral tissues leads to cellular damage and activation of cytokines (tumor necrosis factor- $\alpha$ and nuclear factor-KB) ${ }^{29}$ as well as the renin-angiotensinaldosterone and adrenergic nervous system. These reactions cause insulin resistance ${ }^{30}$, and anabolic-catabolic imbalance. $^{31}$

The CONUT score is calculated from variables reflecting both protein and lipid metabolism, as well as immune function. PNI is similar to the CONUT score but does not include cholesterol, which might be more appropriate in patients with HF as a significant proportion (54\% in our cohort) take statins which cause lower cholesterol levels irrespective of nutritional status. GNRI was the weakest predictor of mortality, perhaps because GNRI includes weight loss. Weight loss is unreliable in patients with HF because of the influence of oedema and the use of diuretics. Assessment of body composition rather than direct weight measurements might be more appropriate. ${ }^{32}$

Amongst the frailty indices, CFS had the strongest prognostic value. The CFS is a more complex tool giving a scored result, whereas AFN and DFI are simple “yes/no” screening tools. CFS is not without its limitations - there is an element of subjectivity, which introduces the possibility of bias.

The management of both frailty and malnutrition is a medical challenge. Exercise therapy, ${ }^{33}$ nutritional supplementation with micronutrients, ${ }^{34}$ calcium and vitamin $\mathrm{D}^{35}, 36$ and multidisciplinary management ${ }^{37}$ may be beneficial. Consensus definitions of malnutrition and frailty are needed so that comparable intervention trials can be designed to study the effect of interventions on outcomes. We have found that amongst the malnutrition indices, PNI had the greatest prognostic value and amonst the frailty scores, CFS had the greatest prognostic value and we would suggest that future work should therefore concentrate on these indices. 


\section{Study limitations}

Firstly, this is a single-centre study conducted in the UK with limited sample size; external validation of our results from authors of other countries with different healthcare and social systems is needed. Secondly, we have only studied six of the large number of screening tools proposed to assess frailty and malnutrition. Thirdly, we have not compared the prognostic value of nutritional screening tools with more complex comprehensive nutritional assessments. ${ }^{38,39}$ Similarly, we have only looked at frailty identification tools rather than comprehensive assessment tools. ${ }^{17,20}$

\section{Conclusion}

Malnutrition and frailty assessed by simple nutritional and frailty indices are very common in patients hospitalised for HF and are powerful predictors of mortality. CFS and PNI should be considered when assessing HF patients to enable identification of individuals at high risk of mortality.

Clinical Perspectives - Malnutrition and frailty assessed by simple nutritional and frailty indices are very common in elderly patients hospitalised for heart failure and are powerful predictors of mortality. The Clinical Frailty Scale and Prognostic Nutritional Index should be used when assessing heart failure patients so as to identify patients at high risk and allow early implementation of tailored treatments or interventions. 
Translational outlook - Future research should focus on standardising definitions of frailty and malnutrition, so that comparable intervention trials can be designed to study the effect of interventions on outcomes in these at-risk heart failure patients. It would also be useful to compare the prognostic value of simple nutritional and frailty screening tools against more complex comprehensive nutritional and frailty assessments in the heart failure population.

\section{Disclosure}

None of the authors has a conflict of interest to disclose.

\section{References}

1 McMurray JJ, Adamopoulos S, Anker SD, et. ESC guidelines for the diagnosis and treatment of acute and chronic heart failure 2012: the task force for the diagnosis and treatment of acute and chronic heart failure 2012 of the European Society of Cardiology. Developed in collaboration with the Heart Failure Association (HFA) of the ESC. Eur Heart J 2012;33:1787-847.

${ }^{2}$ National Heart Failure Audit, April 2012-March 2013. http://wwwuclacuk/nicor/audits/heartfailure/documents/annualreports/hfannual12_ 13pdf(accessed 18.3.2016).

${ }^{3}$ Parmar KR, Xiu PY, Chowdhury MR, Patel E, Cohen M. In-hospital treatment and outcomes of heart failure in specialist and non-specialist services: a retrospective cohort study in the elderly. Open Heart 2015;2:e000095.

${ }^{4}$ Maggioni AP, Dahlström U, Filippatos G, et al. EURObservational Research Programme: regional differences and 1-year follow-up results of the Heart Failure Pilot Survey (ESC-HF Pilot). Eur J Heart Fail 2013;15:808-17.

${ }^{5}$ Walston J, Hadley EC, Ferrucci L, et al. Research agenda for frailty in older adults: Toward a better understanding of physiology and etiology: Summary from the American Geriatrics Society/National Institute on Aging Research Conference on Frailty in Older Adults. $J$ Am Geriatr Soc 2006;54:991-1001

${ }^{6}$ Narumi T, Arimoto T, Funayama A, et al. The prognostic importance of objective nutritional indexes in patients with chronic heart failure. J Cardiol 2013;62:307-313.

${ }^{7}$ Chaudhry SI, McAvay G, Chen S, et al. Risk factors for hospital admission among older persons with newly diagnosed heart failure: findings from the cardiovascular health study. $J$ Am Coll Cardiol 2013;61:635-42. 
${ }^{8}$ Cacciatore F, Abete P, Mazzella F, et al .. Frailty predicts long-term mortality in elderly subjects with chronic heart failure. Eur J Clin Investig 2005;35:723-30.

${ }^{9}$ Lupon J, Gonzalez B, Santaeugenia S, et al. Prognostic implication of frailty and depressive symptoms in an outpatient population with heart failure. Rev Esp Cardiol 2008;61:835-42.

${ }^{10}$ F. Blondé-Cynober, G. Morineau, B. Estrugo, E. Fillie, C. Aussel, J.P. Vincent. Diagnostic and prognostic value of brain natriuretic peptide (BNP) concentrations in very elderly heart disease patients: specific geriatric cut-off and impacts of age, gender, renal dysfunction, and nutritional status. Arch Gerontol Geriatr 2011;52:106-10.

${ }^{11}$ Rahman A, Jafry S, Jeejeebhoy K, Nagpal AD, Pisani B, Agarwala R. Malnutrition and Cachexia in Heart Failure. JPEN J Parenter Enteral Nutr 2015. pii: 0148607114566854. [Epub ahead of print]

12 Otaki M. Surgical treatment of patients with cardiac cachexia. An analysis of factors affecting operative mortality. Chest 1993;105:1347-51.

${ }^{13}$ Rozentryt P, von Haehling S, Lainscak M, et al. The effects of a high-caloric protein- rich oral nutritiaonl supplement in patients with chronic heart failure and cachexia on quality of life, body composition and inflammation markers: a randomized, double-blind pilot study. $J$ Cachex Sarcopenia Muscle 2010;1:35-42.

${ }^{14}$ Lopez D, Flicker L, Dobson A. Validation of the frail scale in a cohort of older Australian women. J Am Geriatr Soc 2012;60:171-173.

15 Subra J, Gillette-Guyonnet S, Cesari M, Oustric S, Vellas B; Platform Team. The integration of frailty into clinical practice: Preliminary results from the Gérontopôle. $J$ Nutr Health Aging 2012;16:714-720.

${ }^{16}$ Ensrud KE, Ewing SK, Taylor BC, et al. Comparison of 2 frailty indexes for prediction of falls, disability, fractures, and death in older women. Arch Intern Med 2008;168:382-389.

${ }^{17}$ Fried LP, Tangen CM, Walston J, et al. Frailty in older adults: Evidence for a phenotype. $J$ Gerontol A Biol Sci Med Sci 2001;56:M146-M156.

${ }^{18}$ Ignacio de Ulíbarri J, González-Madroño A, de Villar NG, et al. CONUT: a tool for controlling nutritional status. First validation in a hospital population. Nutr Hosp 2005; 20:38-45.

${ }^{19}$ O. Bouillanne, G. Morineau, C. Dupont, et al. Geriatric nutritional risk index: a new index for evaluating at -risk elderly medical patients. Am J Clin Nutr 2005;82:777-83.

${ }^{20}$ Rockwood K, Song X, MacKnight C, et al. A global clinical measure of fitness and frailty in elderly people. CMAJ 2005;173:489-495.

21 G.P. Buzby, J.L. Mullen, D.C. Matthews, Hobbs CL, Rosato EF. Prognostic nutritional index in gastrointestinal surgery. Am J Surg 1980;139:160-167.

${ }^{22}$ Alvares-da-Silva MR \& Reverbel da Silveira T. Comparison between handgrip strength, subjective global assessment, and prognostic nutritional index in assessing malnutrition and predicting clinical outcome in cirrhotic outpatients. Nutrition 2005;21:113-7 
${ }^{23}$ J. Woodard, J. Youde, B. Sutton \& S. Bagshaw. Frailty Identification in Acute Medical Admissions. Age Ageing 2014;43:i9.

${ }^{24}$ Acute frailty nework webpage. [Accessed March 3, 2016]; available at: http://www.acutefrailtynetwork.org.uk/uploads/files/1/Resource/SILVER_BOOK_FINAL_M AIN.pdf.

${ }^{25}$ Charlson ME, Pompei P, Ales KL, MacKenzie CR. A new method of classifying prognostic comorbidity in longitudinal studies: development and validation. J Chronic Dis 1987;40:373-383.

${ }^{26}$ Bonilla-Palomas JF, Gamez-Lopez AL, Anguita-Sanchez MP, et al. Impact of malnutrition on long-term mortality in hospitalised patients with heart failure. Rev Esp Cardio 2011;64:752-8.

${ }^{27}$ Aziz EF, Javed F, Pratap B, et al. Malnutrition as assessed by nutritional risk index is associate with worse outcome in patients admitted with acute decompensated heart failure: an ACAP-HF data analysis. Heart Int 2011;6:e2.

${ }^{28}$ Afilalo J, Alexander KP, Mack MJ, et al. Frailty assessment in the cardiovascular care of older adults. J Am Coll Cardiol 2014;63:747-62.

${ }^{29}$ Levine B, Kalman J, Mayer L, Fillit HM, Packer M. Elevated circulating levels of tumor necrosis factor in severe chronic heart failure. N Engl J Med 1990;323:236-241.

${ }^{30}$ Heck PM, Dutka DP. Insulin resistance and heart failure. Curr Heart_Fail Rep 2009;6:8994.

${ }^{31}$ Anker SD, Chua TP, Ponikowski P, et al. Hormonal changes and catabolic/anabolic imbalance in chronic heart failure and their importance for cardiac cachexia. Circulation 1997;96:526-534.

${ }^{32}$ Anker SD, Morley JE. Cachexia: a nutritional syndrome? J Cachexia Sarcopenia Muscle 2015;6:269-71. 
${ }^{33}$ De Vries NM, van Ravensberg CD, Hobbelen JS, Olde Rikkert MG, Staal JB, Nijhuis-van der Sanden MW. Effects of physical exercise therapy on mobility, physical functioning, physical activity and quality of life in community-dwelling older adults with impaired mobility, physical disability and/or multi-morbidity: a meta-analysis. Ageing Res Rev 2012;11:136-49.

${ }^{34}$ Witte KK, Nikitin NP, Parker AC, et al. The effect of micronutrient supplementation on quality-of-life and left ventricular function in elderly patients with chronic heart failure. Eur Heart J 2005;26:2238-44.

${ }^{35}$ Murad MH, Elamin KB, Abu Elnour NO, et al. Clinical review: The effect of vitamin D on falls: a systematic review and meta-analysis. J Clin Endocrinol Metab 2011;96:2997-3006.

${ }^{36}$ Rejnmark L, Avenell A, Masud T, et al. Vitamin D with calcium reduces mortality: patient level pooled analysis of 70,528 patients from eight major vitamin D trials. J Clin Endocrinol Metab 2012;97:2670-81.

${ }^{37}$ Cameron ID, Fairhall N, Langron C, et al. A multifactorial interdisciplinary intervention reduces frailty in older people: randomized trial. BMC Med 2013;11:65.

${ }^{38}$ Aggarwal A, Kumar A, Gregory MP, et al. Nutrition assessment in advanced heart failure patients evaluated for ventricular assist devices or cardiac transplantation. Nutr Clin Pract 2013;28:112-119.

${ }^{39}$ Yamauti AK, Ochiai ME, Bifulco PS, et al. Subjective global assessment of nutritional status in cardiac patients. Arq Bras Cardiol 2006;87:772-777.

\section{Legend to figures.}

Figure 1: Prevalence of frailty in our cohort of patients with acute heart failure according to Derby Frailty Index (frail vs non-frail), Acute Frailty Network (frail vs non-frail) and Clinical frailty scale (CFS) (frail: CSF $>4$ vs non-frail: $\mathrm{CSF} \geq 4$ ).

Figure 2: Prevalence of malnutrition in our cohort of patients with acute heart failure according to Controlling Nutritional Index (CONUT) score (malnourished: CONUT $>4$ vs not malnourished: CONUT $\leq 4)$, Geriatric Nutritional Risk Index (GNRI) (malnourished: 
GNRI $\leq 98$ vs not malnourished: GNRI $>98$ ) and Prognostic Nutritional Index (PNI) (malnourished: $\mathrm{PNI} \leq 38$ vs not malnourished: $\mathrm{PNI}>38$ ).

Figure 3: Relationship between CFS and PNI, $\left(\mathrm{R}^{2}=0.22, \mathrm{p}<0.001\right)$.

Figure 4: Kaplan Meier curves for all-cause mortality by the combined groups of malnutrition (assessed by prognostic nutritional index (PNI), where malnourished: $\mathrm{PNI} \leq 38$ vs not malnourished: PNI >38) and frailty (assessed by clinical frailty scale (CFS), where frail: CSF $>4$ vs non-frail: $\mathrm{CSF} \geq 4$ ). Compared to those not malnourished and not frail, those who were malnourished and frail had a 30-fold higher risk of adverse outcome. 